\title{
A Meta-analysis on efficacy of auto fluorescence in detecting the early dysplastic changes of oral cavity
}

\author{
Nallan C. S. K. Chaitanya, Sunanda Chavva, Elizabeth Surekha, Vedula Priyanka', Mule Akhila, Hari Kiran Ponnuru², \\ Charan Kumar Reddy ${ }^{3}$
}

\begin{abstract}
Background: Light-based detection agents using autofluorescence may be helpful in the detection of early dysplasia, which would otherwise be misdiagnosed as nondysplastic by conventional oral examination (COE) with white light.Visually-enhanced lesion scope (VELscope) is one of such an aid used for the purpose.A meta-analysis was carried out on the sensitivity and specificity ofVELscope that would enable in providing evidence of its usage in oral dysplasia. Materials and Methods: MeSH terms such as auto florescence in oral dysplasia,VELscope, Oral ID, Identifi, in a different medical database such as PubMed, Cochrane, EBSCO, and Google scholar was carried out by four research associates. The total articles available were 242 , of which, 230 were excluded based on strict criteria of randomized control trials and proper design. Finally, only 12 studies were chosen for the present analysis. Of I643 patients from I 2 studies, 1264 patients had undergone the autofluorescence examination after the COE. Only 774 patients have shown the loss of fluorescence with VELScope examination and 487 had retained the fluorescence. Biopsy was performed on II 76 patients after the autofluorescence examination in the areas where there was the loss of fluorescence. The available data were subjected to software Review Manager for analysis. Results and Discussion: Of the II studies analyzed, majority of them showed that the autofluorescence device were sensitive enough $>0.70$; however, the values of sensitivity and specificities varied significantly. With the VELscope the diagnostic performance of the inexpert examiner was not improved, obtaining a sensitivity of 0.40 ( $95 \%$ of confidence interval [Cl]: 0.406-0.773) and a specificity of 0.80 (95\% Cl: 0.6 I4-0.923). Conclusion: The new technique may help as an adjunct to histopathology in detecting the dysplasia initially and stop further progression to the carcinoma.
\end{abstract}

Key words: Autofluorescence, meta-analysis, visually enhanced lesion scope

\section{Introduction}

Oral cancer is the sixth most common cancer worldwide and is mostly preceded by the oral potentially malignant disorders (OPMDs) such as leukoplakia, oral submucous fibrosis, erythroplakia, and lichen planus. In the course of its progression, multiple changes at the cellular and at the corresponding tissue level occur, that pose a high risk of malignant transformation. OPMDs, hence are regarded as histological markers indicating the high risk of oral cancer occurrence. These OPMDs are recognized by early dysplastic changes they pose histologically and are thus helpful in early diagnosis of the changes that are attributed to the rise in oral carcinoma. Conventional oral examination (COE) and biopsy allow early detection of premalignant and cancerous conditions. ${ }^{[1]}$ The current standard of clinical examination is by white light which identifies the OPMDs and other mucosal disorders but not overt lesions which are not detectable to white light or $\mathrm{COE}^{[2]}$

Various techniques have been developed to detect the early mucosal changes, such as Vital staining procedures (Toluidine Blue, Methylene Blue, Rose Bengal dye and Lugol's Iodine), cytopathological examination (Oral exfoliative cytology, oral brush biopsy, micro nuclei analysis, DNA ploidy, liquid-based cytology, tumor markers, and biomarkers $)^{[3]}$ and light-based techniques, which include chemiluminescence (ViziLite, ViziLite plus, MicroLux TM/DM) and autofluorescence imaging visually-enhanced lesion scope (VEL scope, Oral ID). Autofluorescence is a technique that is based on the principle that the naturally occurring fluorophores located in the mucosa and submucosa show fluorescence at the green spectral range

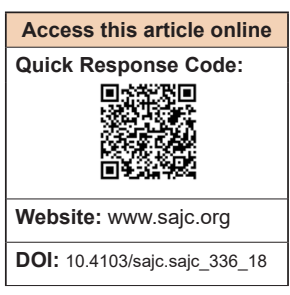

Department of Oral Medicine and Radiology, Panineeya Mahavidyalaya Institute of Dental Sciences and Research Centre, Departments of 'Oral Pathology and Microbiology and ${ }^{3}$ Oral Medicine and Radiology, Panineeya Institute of Dental Sciences, Hyderabad, ${ }^{2}$ Department of Conservative Dentistry and Endodontics, Mamata Dental College, Khammam, Telangana, India

Correspondence to: Dr. Nallan C.S.K. Chaitanya, E-mail: nallanchaitanya@gmail.com when irradiated between the wavelengths of 375 and $440 \mathrm{~nm}$. Various studies have been done to evaluate the effectiveness of these techniques in detecting the early dysplastic lesions. ${ }^{[4]}$ Even though the oral cavity is accessible for visual examination, the different clinical manifestations presented by various OPMDs make it difficult to detect the lesion in its earlier stages. This can be overcome by the phenomenon of autofluorescence which makes it possible to evaluate suspicious lesions for any dysplastic changes, thereby decreasing the morbidity of the patient.

VEL scope is a noninvasive, wireless, simple device that works on the principle of auto fluorescence, which emits harmless, bright blue light, helpful in inspecting the oral cavity. VELscope is sensitive to abnormal tissue changes. Normal mucosal tissue fluoresces in distinct patterns, that may be visibly disrupted when tissue undergoes an abnormal change, such as when associated with dysplasia. ${ }^{[5]}$ The VELscope has been approved by the US Food and Drug Administration as an adjunct to traditional oral examination to enhance the visualization of oral mucosal abnormalities, and its clinical use appears to be growing. ${ }^{[6]}$

Identafi 3000 device consists of three light sources-white light for regular illumination, a violet light that excites fluorescence at $405 \mathrm{~nm}$ for tissue absorption, and a green-amber light at $545 \mathrm{~nm}$ for tissue reflectance. ${ }^{[7]}$

The present study was intended to evaluate the effectiveness of the VELscope in detecting the dysplastic changes at an early stage. This meta-analysis was performed to analyze various studies in the literature using autofluorescence as an adjunctive tool in objectively identifying the site of dysplasia in various OPMDs conditions.

This is an open access journal, and articles are distributed under the terms of the Creative Commons Attribution-NonCommercial-ShareAlike 4.0 License, which allows others to remix, tweak, and build upon the work non-commercially, as long as appropriate credit is given and the new creations are licensed under the identical terms.

For reprints contact: reprints@ medknow.com

How to cite this article: K. Chaitanya NC, Chavva S, Surekha E, Priyanka V, Akhila M, Ponnuru HK, et al. A meta-analysis on efficacy of auto fluorescence in detecting the early dysplastic changes of oral cavity. South Asian J Cancer 2019;8:233-6. 


\section{Materials and Methods}

The present study evaluates randomized controlled trials using the principle of auto fluorescence in detecting the site of dysplasias in OMPD. Databases such as Pub Med, Cochrane, Research Gate, and Google Scholar were searched using the terms Autofluorescence, VELscope, Oral ID, Identafi 3000 and articles related to noninvasive techniques useful for diagnosis that are published from 2000 to 2017 are referred.

Exclusion criteria included-articles from foreign languages other than English, case reports, cohort studies, articles with only abstract being available and review articles. Studies were assessed by four researchers who contributed for literature search, article retrieval and analysis of same in accordance with the protocol of the study. The articles satisfying the inclusion criteria, proper design, and correct conclusions pertaining to usage of autofluorescence principle in the detection of the early precancerous lesions were extracted and used for meta-analysis.

Data selection, recovery, and analysis

Data were selected by four researchers, where the primary researcher had collected the information concerning autofluorescence principle that is used to detect the site of biopsy within the potentially malignant disorders, the second researcher had analyzed on various devices that used this principle, only VELscope was retrieved as most commonly used device. The third researcher had sought for exclusive VELscope related articles retrieved from the second research assistant. All research assistants reported to primary research worker who was the ultimate authority.

A total of 7735 articles were collected from the databases. After completely assessing the eligibility criteria, 7493 were excluded as they were in a foreign language, only with abstract, irrelevant interventions and with improper citations. Among these 242 articles, 230 were excluded owing to their improper designs, duplicates, and reviews. A total of 12 studies were chosen that were apt to the present analysis. A total of 1643 patients were included in the analysis of all 12 studies and 1264 patients had undergone the autofluorescence examination after the COE. A total of 774 patients have shown the loss of fluorescence with VELScope examination. A total of 487 have retained the fluorescence. A biopsy was done in 1176 patients after the auto fluorescence examination in the areas where there is a loss of fluorescence.

Thus, obtained data were tabulated having characteristics of gender, loss of fluorescence, retained fluorescence, histopathology, and the grade of dysplasia. Data, thus extracted were statistically evaluated using the software Review Manager (RevMan) [Computer program]. Version 5.3. Copenhagen: The Nordic Cochrane Centre, The Cochrane Collaboration, 2014.

\section{Results}

\section{Statistical output}

This meta-analysis was carried out to evaluate the efficacy of autofluorescence using devices with respect to their sensitivity and specificity and also the ability to identify the site of biopsy in the potentially malignant disorders. Confidence interval (CI) $95 \%$ with $P$ value significance taken at $<0.05$ was taken as criteria for obtaining the statistical analysis.
A study by Jané-Salas et al., ${ }^{[8]}$ was excluded from the analysis because it consisted of variable diagnosis given by

a. Expert (made by an oral medicine specialist)

b. Inexpert (made by a general dentist) and

c. VELscope assisted inexpert diagnosis, and the values for sensitivity and specificity were 1 (95\% CI: $0.884-1)$ for expert diagnosis.

Performance of inexpert diagnosis has reduced to 0.53 sensitivity (95\% CI: $0.343-0.717)$ and 0.83 specificity (95\% CI: $0.653-0.944)$. With the use of VELscope the diagnostic performance of the inexpert examiner was not improved, obtaining a sensitivity of 0.40 (95\% CI: 0.406-0.773) and a specificity of 0.80 (95\% CI: $0.614-0.923)$ and concluded that no clinical benefits were obtained using this VELscope.

The receiver operating characteristic curve [Figure 1]. It is defined on a plot of test sensitivity as they coordinate versus its 1-specificity or false-positive rate (FPR) as the $\mathrm{x}$ coordinate. Thus, it showed the sensitivity and specificity of all the studies which were included in the analysis.

Out of the eleven studies, the majority of them showed that the autofluorescence device was sensitive enough; however, the values between the sensitivity and specificities varied significantly between the studies. The lowest sensitivity (i.e., 0.30) was from the study conducted by the Farah et al..$^{[5]}$ in 112 patients with oral mucosal white or mixed red/white lesion that were clinically suspicious and concluded that autofluorescence to be of use at aiding the visualization of potentially malignant, malignant, and inflammatory conditions; however, it could not differentiate between them.

The two studies, by the Rana et al. ${ }^{[0]}$ and the Scheer et al., ${ }^{[10]}$ were more accurate with the sensitivity values of 1 . The study conducted by the Scheer et al., showed the $100 \%$ sensitivity of the device and specificity of $80.8 \%$ with a positive predictive value of $54.5 \%$ and negative predictive value of $100 \%$ and they concluded that VELscope which used blue light of wavelength $430 \mathrm{~nm}$, could assist identification malignant and OPMDs from normal mucosa in high-risk patients but does not discriminate benign lesions from malignant and premalignant lesions.

The two studies, by Lane et al. ${ }^{[1]}$ and Hanken et al. ${ }^{[12]}$ showed the sensitivity values of 0.98 . The study conducted by Lane et al. ${ }^{[1]}$ showed the specificity of 1.00 and the study conducted by Hanken et al.,$^{[12]}$ had showed the different sensitivity values for only white, white light and VELScope, and their differences and the values were $75.9 \%, 97.9 \%$, and $22 \%$, respectively, and concluded that the technique of autofluorescence can be used to identify the site of biopsy and for marginal delineation during the surgical procedures. The study conducted by Felix peter Koch et al. ${ }^{[13]}$ showed the sensitivity value of 0.94 having a CI $(0.80,0.99)$. The study

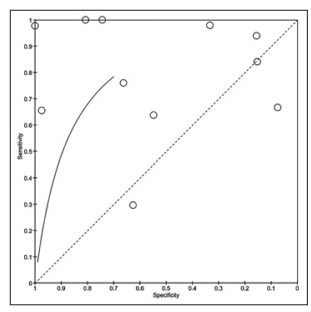

Figure 1: Receiver operating characteristic curve and various studies within and above the boundaries of the curve

South Asian Journal of Cancer V Volume 8 Issue 4 October-December 2019 
conducted by Felix peter Koch et al. ${ }^{[13]}$ showed the sensitivity value of 0.94 having a CI $(0.80,0.99)$ and specificity of 0.16 . It also showed a positive predictive value of $45 \%$ and negative predictive value of $77 \%$. Thus, it concluded that autofluorescence examination could not differentiate between the benign and malignant oral lesions.

A study by Awan et al., ${ }^{[2]}$ which consisted a total of 126 patients with oral mucosal lesions were taken, and all were subjected to COE followed by VELscope and biopsy among these 44 had oral epithelial dysplasia. The sensitivity and specificity of autofluorescence for the detection of dysplastic lesions were 0.84 with CI value of $(0.70$, and 0.93$)$ and 0.15 . He concluded that VELscope was unable to discriminate high-risk from low-risk lesions.

The study conducted by Ganga et al., ${ }^{[14]}$ revealed the sensitivity and specificity values of $76 \%$ and $66.29 \%$, respectively, whereas the positive and negative predictive values were $24.36 \%$ and $95.08 \%$, respectively. The high negative predictive value is due to high false-positive results and low specificity rate and false-negative results limited the sensitivity and concluded that VELscope alone cannot replace the COE, biopsy and histopathology evaluation, which remain the gold standard.

The other studies conducted by Bhatia et al., ${ }^{[15]}$ Paderni et al., ${ }^{[16]}$ and McNamara et al., ${ }^{[17]}$ have shown the sensitivity and specificity values $0.64,0.66,0.67$ and $0.55,0.97,0.07$, respectively. They concluded that autofluorescence using devices cannot totally replace the $\mathrm{COE}$ and histopathological evaluation but can be used as a complementary device during diagnosing and monitoring the different steps.

Paired forest plots [Figure 2] are presented together for sensitivity and other for specificity. The square boxes of the studies, toward the right of the graph, indicate an increase in the sensitivity/specificity and towards left indicate a decrease in the sensitivity/specificity. The maximum and minimum values taken as 0 and 1 . The studies conducted by the Scheer et al. (2011) and Rana et al. (2012) had shown their at value 1 , indicating that autofluorescence technique can be used detect the early dysplastic changes. The studies conducted by Felix peter koch et al. (2011) and Hanken et al. (2013) have the boxes at 0.94 and 0.98 , respectively, supporting the autofluorescence technique. A study by Farah et al. (2011) has the value at the left side of the graph, indicating that this technique did not help in detecting the dysplastic changes. Other studies show their values dispersed, but more toward right indicating that autofluorescence technique can be used in detecting the early dysplastic changes. A study by Lane et al. (2006), have the sensitivity and specificity value toward the extreme left of the graph concluding that VELscope can be used as an adjunct to COE screening to increase the sensitivity of white-light screening alone but cannot reduce the

\begin{tabular}{|c|c|c|c|c|c|c|c|c|}
\hline Study & TP & FP & & & Sensitivity (95\% Cl) & Specificity $(95 \%$ Cl) & Sensitivity $(95 \% \mathrm{Cl})$ & Specificity $(95 \%$ \\
\hline Awan et al 2011 & 37 & 61 & 7 & 11 & $0.84[0.70,0.93]$ & $0.15[0.08,0.26]$ & & \\
\hline Bhatia 2016 & 95 & 335 & 54 & & $0.64[0.55,0.71]$ & $0.55[0.43,0.66]$ & & \\
\hline C Paderni et al 2011 & 19 & 3 & 101 & 15 & $0.66[0.46,0.82]$ & $0.97[0.93,0.99]$ & & $=$ \\
\hline Farah et al 2011 & & & 19 & 57 & $0.30[0.14,0.50]$ & $0.63[0.52,0.73]$ & & \\
\hline Felix peter koch et al 2011 & & & & 7 & $0.94[0.80,0.99]$ & $0.16[0.06,0.29]$ & & \\
\hline Ganga et al 2017 & & 59 & & & $0.76[0.55,0.91]$ & $0.66[0.59,0.73]$ & & \\
\hline Hanken et al 2013 & 47 & 8 & 1 & 4 & $0.98[0.89,1.00]$ & $0.33[0.10,0.65]$ & & \\
\hline Lane et al 2006 & 43 & 0 & 1 & 6 & $0.98[0.88,1.00]$ & $1.00[0.54,1.00]$ & & \\
\hline Mc Namara 2012 & & 37 & 1 & 3 & $0.67[0.09,0.99]$ & $0.07[0.02,0.20]$ & & \\
\hline Rana 2012 & 63 & 30 & & 87 & $1.00[0.54,1.00]$ & $0.74[0.65,0.82]$ & & \\
\hline Scheer 2011 & 12 & & & 42 & $1.00[0.74,1.00]$ & $0.81[0.67,0.90]$ & & F1 \\
\hline
\end{tabular}

Figure 2: Various studies with their sensitivity and specificity values

South Asian Journal of Cancer Volume 8 - Issue 4 October-December 2019 specificity. The studies done by other authors show their values of specificity dispersed all over the graph indicating publication bias of the studies.

\section{Discussion}

Oral squamous cell carcinoma (OSCC) is the sixth most cancer worldwide, and the prevalence is more in some countries such as Pakistan, India, and Bangladesh. ${ }^{[18]}$ Even after the numerous advancements in the treatment modality, the survival rate has only remained $50 \%$, this is due to misdiagnosing the disease in its early stages, and many oral lesions undergo biopsy only after they express the malignant features. Therefore, the early diagnosis of the disease is important for the better survival and to improve the quality of life postmalignancy.

To diagnose this OSCC in the early stages, the autofluorescence technique was used in the early 1920's. ${ }^{[18]}$ This technique uses an extrinsic light to excite the endogenous fluorophores present in the oral mucosa such as amino acids, metabolic products, and structural proteins. The oral epithelium consists of nicotinamide adenine dinucleotide, flavin adenine dinucleotide, and collagen cross-links in the stroma. The fluorophores absorb the extrinsic light which consists of the high energy photons and emits the lower energy photons and each fluorophore is associated with a particular excitation and emission wavelengths. When excited with a specific wavelength, the normal mucosa emits the pale green auto fluorescence when vowed through the specific narrow band filters, whereas the tissue with the dysplastic cells shows the loss of fluorescence or the darker areas when compared to normal due to the disruption in the distribution of the fluorophores. ${ }^{[15]}$ Even the inflammatory conditions may result in an increased blood supply to the lesion, and the increased hemoglobin content may absorb light and cause the loss of fluorescence mimicking dysplasias.

The present analysis included 11 studies, in which the participants were diagnosed with at least one or more oral mucosal lesions with COE.

In a study conducted by Rana et al., ${ }^{[9]}$ VELscope in comparison of COE markedly increased the sensitivity of detecting dysplasia with a value of $17 \%-100 \%$. However, majority of the lesions did not undergo histopathological assessment to confirm the presence or absence of the dysplasia leading to bias. A study conducted by Scheer et al., ${ }^{[10]}$ high sensitivity value of $100 \%$ and limited specificity value $80.8 \%$ were obtained. However, VELscope examination identified all the dysplastic and invasive carcinomas by the loss of fluorescence.

The study conducted by Hanken et al. ${ }^{[12]}$ concluded that VELscope has a higher sensitivity (22.0\%), and lower specificity $(8.4 \%)$. Furthermore, it is more promising than $\mathrm{COE}$ in detecting precursor oral malignant lesions. Another study having a high sensitivity of device was conducted by Lane et al. ${ }^{[11]}$ using histology as the gold standard the device achieved a sensitivity of $98 \%$ in discriminating normal mucosa from severe dysplasia or carcinoma in situ high sensitivity in identifying the dysplasia. However, VELscope showed the loss of fluoroscence in benign and the inflammatory conditions, which showed the high specificity of the device.

McNamara et al. ${ }^{[17]}$ concluded that COE was more valid than autofluorescence examination with VELscope in routine screening for potentially malignant disorders and believed 
that careful, COE of the entire oral cavity on a regular basis remains the gold standard for early detection of OPMD.

Farah et al..$^{[5]}$ conducted a study in which he concluded that the combination of COE and VELscope is more beneficial in identifying the dysplastic changes when compared to only $\mathrm{COE}$ or VELscope (diascopic fluorescence) and only the VELscope examination may not be useful.

The efficacy of a diagnostic test is dependent on its sensitivity and specificity values. Overall, seven studies out of eleven have shown the sensitivity values $>0.70$ and with varied specificity values.

\section{Conclusion}

Identifying the oral lesions before transforming to neoplasia is important to increase the survival rate of the patient and for a good prognosis. However, only COE may not be sufficient to detect the lesions in the early stages, and hence, the new adjunct techniques may help the clinicians to detect the dysplastic changes initially and stop further progression of the disease to the OSCC. Autofluorescence using devices may be used as adjunct to find the exact location of the biopsy in altered mucosal conditions. Due to high FPRs the use of these devices is limited. However, the COE followed by biopsy of the lesion will remain the gold standard of the detection of the lesions.

\section{Acknowledgment}

We would like to thank the staff of the Department of Oral Medicine and Radiology for extending their support during the present analysis.

\section{Financial support and sponsorship}

Nil.

\section{Conflicts of interest}

There are no conflicts of interest.

\section{References}

1. Shield KD, Ferlay J, Jemal A, Sankaranarayanan R, Chaturvedi AK, Bray F, et al. The global incidence of lip, oral cavity, and pharyngeal cancers by subsite in 2012. CA Cancer J Clin 2017;67:51-64

2. Awan KH, Morgan PR, Warnakulasuriya S. Evaluation of an autofluorescence based imaging system (VELscope ${ }^{\mathrm{TM}}$ ) in the detection of oral potentially malignant disorders and benign keratoses. Oral Oncol 2011;47:274-7.

3. Singla S, Verman A, Goyal S, Singla I, Shetty A. A conventional and advanced diagnostic tools in oral cancer with emphasis on role of dentist and early detection. J Postgrad Med Educ Res 2017;51:128-33.

4. Nagi R, Reddy-Kantharaj YB, Rakesh N, Janardhan-Reddy S, Sahu S. Efficacy of light based detection systems for early detection of oral cancer and oral potentially malignant disorders: Systematic review. Med Oral Patol Oral Cir Bucal 2016;21:e447-55.

5. Farah CS, McIntosh L, Georgiou A, McCullough MJ. Efficacy of tissue autofluorescence imaging (VEL scope) in the visualization of oral mucosal lesions. Head Neck 2012;34:856-62.

6. Shin D, Vigneswaran N, Gillenwater A, Richards-Kortum R. Advances in fluorescence imaging techniques to detect oral cancer and its precursors. Future Oncol 2010;6:1143-54.

7. Bhatia N, Lalla Y, Vu AN, Farah CS. Advances in optical adjunctive AIDS for visualisation and detection of oral malignant and potentially malignant lesions. Int J Dent 2013;2013:194029.

8. Jané-Salas E, Blanco-Carrión A, Jover-Armengol L, López-López J. Autofluorescence and diagnostic accuracy of lesions of oral mucosa: A pilot study. Braz Dent J 2015;26:580-6.

9. Rana M, Zapf A, Kuehle M, Gellrich NC, Eckardt AM. Clinical evaluation of an autofluorescence diagnostic device for oral cancer detection: A prospective randomized diagnostic study. Eur J Cancer Prev 2012;21:460-6

10. Scheer M, Neugebauer J, Derman A, Fuss J, Drebber U, Zoeller JE, et al. Autofluorescence imaging of potentially malignant mucosa lesions. Oral Surg Oral Med Oral Pathol Oral Radiol Endod 2011;111:568-77.

11. Lane PM, Gilhuly T, Whitehead P, Zeng H, Poh CF, Ng S, et al. Simple device for the direct visualization of oral-cavity tissue fluorescence. J Biomed Opt 2006; 11:024006.

12. Hanken H, Kraatz J, Smeets R, Heiland M, Assaf AT, Blessmann M, et al. The detection of oral pre- malignant lesions with an autofluorescence based imaging system (VELscope ${ }^{T M}$ ) - A single blinded clinical evaluation. Head Face Med 2013;9:23.

13. Koch FP, Kaemmerer PW, Biesterfeld S, Kunkel M, Wagner W. Effectiveness of autofluorescence to identify suspicious oral lesions - A prospective, blinded clinical trial. Clin Oral Investig 2011;15:975-82.

14. Ganga RS, Gundre D, Bansal S, Shirsat PM, Prasad P, Desai RS, et al. Evaluation of the diagnostic efficacy and spectrum of autofluorescence of benign, dysplastic and malignant lesions of the oral cavity using VELscope. Oral Oncol 2017;75:67-74.

15. Bhatia N, Matias MA, Farah CS. Assessment of a decision making protocol to improve the efficacy of VELscope ${ }^{\mathrm{TM}}$ in general dental practice: A prospective evaluation. Oral Oncol 2014;50:1012-9.

16. Paderni C, Compilato D, Carinci F, Nardi G, Rodolico V, Lo Muzio L, et al. Direct visualization of oral-cavity tissue fluorescence as novel aid for early oral cancer diagnosis and potentially malignant disorders monitoring. Int J Immunopathol Pharmacol 2011;24:121-8.

17. McNamara KK, Martin BD, Evans EW, Kalmar JR. The role of direct visual fluorescent examination (VELscope) in routine screening for potentially malignant oral mucosal lesions. Oral Surg Oral Med Oral Pathol Oral Radiol 2012;114:636-43.

18. Awan KH, Patil S. Efficacy of autofluorescence imaging as an adjunctive technique for examination and detection of oral potentially malignant disorders: A systematic review. J Contemp Dent Pract 2015;16:744-9. 